\title{
Beamformer Design for Interference Alignment Using Reweighted Frobenius Norm Minimization
}

\author{
Gokul Sridharan and Wei Yu \\ The Edward S. Rogers Sr. Department of Electrical and Computer Engineering \\ University of Toronto, Toronto, ON, M5S 3G4, Canada \\ Email:gsridharan@comm.utoronto.ca,weiyu@comm.utoronto.ca
}

\begin{abstract}
This paper proposes an algorithm to compute the uplink transmit beamformers for linear interference alignment in MIMO cellular networks without symbol extensions. In particular, we consider interference alignment in a network consisting of $G$ cells and $K$ users/cell, having $N$ and $M$ antennas at each base station (BS) and user respectively. Using an alternate interpretation of the conditions for interference alignment, we frame the problem of finding aligned transmit beamformers in the uplink as an optimization problem to minimize the rank of a set of interference matrices subject to affine constraints. The interference matrix of a BS consists of all the interfering vectors at that BS. The proposed algorithm approximates rank using the weighted Frobenius norm and iteratively updates the weights so that the weighted Frobenius norm is a close approximation of the rank of the interference matrix. A crucial aspect of this algorithm is the weight update rule that guides the algorithm towards aligned beamformers. We propose a novel weight update rule that discourages the algorithm from converging to local minima that do not generate the requisite number of interference free dimensions. The proposed algorithm is computationally efficient since it only requires solving a simple quadratic program in each iteration. Simulation results indicate much faster convergence to aligned beamformers when compared to algorithms of similar complexity.
\end{abstract}

\section{INTRODUCTION}

Linear beamforming techniques for interference mitigation are of significant interest in MIMO cellular networks where increasing density along with new backhaul enhancements have necessitated and enabled coordinated interference management. In this context interference alignment has emerged as a key concept in addressing interference in such networks. In contrast to asymptotic interference alignment schemes [1], [2] that typically require decomposition of multi-antenna nodes and infinite symbol extensions, linear beamforming schemes are simpler to implement and therefore more relevant in practice. In this work we develop an algorithm to design aligned transmit and receive beamformers to achieve a given number of degrees of freedom (DoF) in a $G$-cell, $K$-user/cell network with $M$ antennas at each user and $N$ antennas at each base station a $(G, K, M, N)$ network.

Our interest in designing algorithms for interference alignment is threefold. First, in cooperative cellular networks that operate in an interference-limited regime, these beamformers identify regions in the optimization landscape where interference is significantly mitigated. Second, while there exist several algebraic-geometry-based techniques that establish feasibility of interference alignment, iterative algorithms are still typically necessary to design the beamformers that achieve the requisite number of DoF. While several iterative algorithms have been proposed to design aligned beamformers [3]-[9], they are not always guaranteed to converge to a set of aligned beamformers that achieve the requisite number of DoF. Third, constructive approaches to design aligned beamformers such as subspace alignment chains [10] are not yet available for a broad class of $(G, K, M, N)$ networks. Thus, iterative algorithms for designing aligned beamformers are very much of interest to the research community.

Several iterative algorithms are available to design beamformers for interference alignment [3]-[9]. In [3], an iterative algorithm for the MIMO interference channel based on minimizing the sum of interference power at all the receivers is proposed, and this algorithm is extended to MIMO cellular networks in [5]. While the algorithms of [3] and [5] are known to converge, they typically need several thousand iterations (although, the per-iteration complexity is low as they only require computing a small number of eigenvalue decompositions per iteration). In [6], a rank constrained rank minimization framework for finding linear beamformers for interference alignment is proposed. Rank is approximated using the nuclear norm, which is suited for inducing sparsity. In [8], [9], [11] a reweighted nuclear norm approach to finding aligned beamformers is proposed where the rank of a certain interference matrix is minimized. Due to the nuclear norm approximation, the algorithms of [6], [8], [9], [11] involve solving a series of semidefinite programs. These algorithms are more computationally intensive than the algorithms of [3], [5], but they require fewer iterations.

In this work, we develop a computationally efficient iterative algorithm for designing aligned beamformers for MIMO cellular/interference networks. We focus on linear interference alignment without symbol extensions and assume the channels to be generic. Using an alternate set of conditions for interference alignment developed in [11], we cast the problem of finding aligned beamformers as a rank minimization problem subject to linear constraints. Using this framework, we develop an iterative weighted-Frobenius-norm minimization algorithm to obtain a set of aligned beamformers. A crucial aspect of this algorithm is a novel reweighting method that takes advantage of the knowledge of the achievable DoF of the network to steer the optimization procedure away from undesirable local optimum solutions. This algorithm is inspired by a similar framework developed for rank minimization in the context of the matrix completion problem [12]. Every iteration of this new algorithm only requires solving an unconstrained quadratic 
program, which are computationally easy to do. Further, unlike existing algorithms, this algorithm does not require alternately optimizing transmit and receive beamformers as the only variables of optimization are transmit beamformers in the uplink.

\section{System MOdEL}

Consider the uplink of a cellular network consisting of $G$ interfering cells with $K$ users per cell. Each user is assumed to have $M$ antennas and each BS is assumed to have $N$ antennas. Let the channel from the $k$ th user in the $g$ th cell to the $i$ th base station (BS) be denoted as the $N \times M$ matrix $\mathbf{H}_{(g k, i)}$. We assume all channels to be generic. All channels are assumed to be known perfectly and available at a central location. In the uplink, let $\mathbf{x}_{g k}$ denote the $M \times 1$ signal vector transmitted by the $k$ th user in the $g$ th cell. This transmit signal vector is formed using a $M \times d_{g k}$ linear transmit beamforming matrix $\mathbf{V}_{g k}$ and received using a $N \times d_{g k}$ receive beamforming matrix $\mathbf{U}_{g k}$, where $d_{g k}$ represents the number of transmitted data streams of the $k$ th user in the $g$ th cell. The received signal after being processed by the receive beamforming matrix $\mathbf{U}_{g k}$ at the $g$ th BS can be written as

$$
\mathbf{U}_{g k}^{H} \mathbf{y}_{g}=\sum_{i=1}^{G} \sum_{j=1}^{K} \mathbf{U}_{g k}^{H} \mathbf{H}_{(i j, g)} \mathbf{V}_{i j} \mathbf{s}_{i j}+\mathbf{U}_{g k}^{H} \mathbf{n}_{g},
$$

where $\mathbf{s}_{i j}$ is the $d_{i j} \times 1$ symbol vector transmitted by the $j$ th user in $i$ th cell and $\mathbf{n}_{g}$ is the $N \times 1$ vector representing circular symmetric additive white Gaussian noise $\sim \mathcal{N}(\mathbf{0}, \mathbf{I})$. While the framework developed in this paper is applicable for any set of $d_{g k} \mathrm{~s}$ that constitute a proper and feasible system, we restrict our focus to the symmetric case where $d_{g k}=d \forall g, k$. The downlink received signal is defined similarly.

We denote the space occupied by interference at the $g$ th BS as the column span of a matrix $\mathbf{R}_{g}$ formed using the column vectors from the set $\left\{\mathbf{H}_{(i j, g)} \mathbf{v}_{i j l}: i \in\{1,2, \ldots, G\}, j \in\right.$ $\{1,2, \ldots, K\}, l \in\{1,2, \ldots, d\}, i \neq g\}$, where we use the notation $\mathbf{v}_{i j l}$ to denote the $l$ th beamformer associated with the $j$ th user in the $i$ th cell.

\section{RANK Minimization APPROACH}

The conditions for linear interference alignment when symbol extensions over time or frequency are not allowed can be stated as follows [13]:

$$
\begin{aligned}
\mathbf{U}_{g k}^{H} \mathbf{H}_{i j, g} \mathbf{V}_{i j} & =\mathbf{0} \forall(i, j) \neq(g, k) \\
\operatorname{rank}\left(\mathbf{U}_{g k}^{H} \mathbf{H}_{g k, g} \mathbf{V}_{g k}\right) & =d \forall(g, k) .
\end{aligned}
$$

Counting the number of equations and variables involved in the conditions gives a preliminary check on the feasibility of satisfying (2) and (3). When the number of variables exceed the number of equations, the system is said to be proper. A $(G, K, M, N)$ network where $d$ DoF/user are desired is proper if $(M+N) \geq(G K+1) d$ [5], [13]. While not all proper systems are feasible [10], improper systems have been shown to be almost surely infeasible [14]. In this paper, we only consider proper systems that are known to be feasible. As stated earlier, feasibility of certain proper systems can be established through certain non-constructive techniques in algebraic geometry.
We briefly state an alternative reformulation of the conditions for interference alignment introduced in [11]. Conditions (2) and (3) can be alternately stated as

$$
\begin{array}{r}
\operatorname{rank}\left(\mathbf{R}_{g}\right) \leq N-K d \forall g, \\
\operatorname{rank}\left(\mathbf{V}_{g k}\right)=d \forall g, k .
\end{array}
$$

This reformulation follows from the fact that when interference spans no more than $N-K d$ dimensions, generic channels ensure that the intersection between useful signal subspace $\left(\operatorname{span}\left(\left\{\mathbf{H}_{(g k, g)} \mathbf{V}_{g k}\right\}_{k=1}^{K}\right)\right)$ and interference subspace $\left(\operatorname{span}\left(\mathbf{R}_{g}\right)\right)$ is almost surely zero dimensional. This allows us to replace (2) with a rank constraint on the interference subspace (4), which ensures that sufficient dimensions are available at each BS for the desired signals. In addition, since generic channel matrices are almost full rank, condition (3) is satisfied as long as $\operatorname{rank}\left(\mathbf{V}_{g k}\right)=d$. This gives rise to condition (5) in the reformulation. Once a set of transmit precoders $\left\{\mathbf{V}_{g k}\right\}$ that satisfy the above conditions are designed, designing the receive filters is then straightforward. We collectively refer to the set of $\left\{\mathbf{V}_{g k}\right\}$ as $\mathbf{V}$.

Since we need to design transmit beamformers that satisfy conditions (4) and (5), it is natural to pose the problem of finding these beamformers as a feasibility problem, as given below:

$$
\begin{array}{cl}
\operatorname{minimize} & 1 \\
\text { subject to } & \operatorname{rank}\left(\mathbf{R}_{g}\right) \leq N-K d \forall g, \\
& \operatorname{rank}\left(\mathbf{V}_{g k}\right)=d \forall(g, k) .
\end{array}
$$

While the rank constraint on $\mathbf{V}_{g k}$ is easily handled by imposing the condition $\mathbf{V}_{g k}(1: d, 1: d)=\mathbf{I} \forall g, k$, handling the rank constraint on $\mathbf{R}_{g}$ is not straightforward. In this paper we eventually use a surrogate function to approximate the rank function. However, before doing so, we first propose a rank minimization reformulation of the above problem. This is because it is easier to handle the rank function in the objective rather than in a constraint.

\section{Min-Max Rank Formulation}

To overcome the difficulty of handling rank constraints, we reformulate the feasibility problem as a minimax optimization problem where we minimize the maximum rank of the matrices $\mathbf{R}_{1}, \mathbf{R}_{2}, \ldots, \mathbf{R}_{G}$. The minimax optimization problem can be stated as

$$
\begin{aligned}
\min _{\mathbf{V}} \max _{g \in\{1,2, \ldots, G\}} & \operatorname{rank}\left(\mathbf{R}_{g}\right) \\
\text { subject to } & \mathbf{V}_{g k}(1: d, 1: d)=\mathbf{I} \forall(g, k) \\
& \mathcal{A}_{g}(\mathbf{V})=\mathbf{R}_{g} \forall g,,
\end{aligned}
$$

where $\mathcal{A}_{g}(\mathbf{V})=\left(\mathbf{R}_{g}\right)$ captures the linear relationship between $\mathbf{V}$ and $\mathbf{R}_{g}$. Since we assume the given system to be proper and feasible, it is clear that the global optimum of this optimization problem is no more than $N-K d$. Further, it can be seen that any set of beamformers in the domain of this optimization problem that ensures the objective is no more than $N-K d$ constitutes a set of aligned solutions. In order to apply standard optimization techniques to solve this optimization problem, we first approximate rank using a surrogate function. Several 
surrogate functions that are well suited for rank minimization problems are known, and they include functions such as nuclear norm (convex envelope of rank), Schatten- $p$ function [12], [15], $\log (\operatorname{det}(\cdot))$ and $-\operatorname{tr}(\operatorname{inv}(\cdot))$ [16]-[18]. While nuclear norm, $\log (\operatorname{det}(\cdot))$ and $-\operatorname{tr}(\operatorname{inv}(\cdot))$ approximations lead to solving a sequence of semidefinite programs, Schatten- $p$ function requires solving a series of quadratic programs where a weighted Frobenius norm is iteratively minimized.

With computational complexity in mind and drawing inspiration from the reweighted Frobenius norm minimization approach developed in [12] for the affine-constrained rank minimization problem, we approximate rank using a series of weighted Frobenius norms. Note that the square of the Frobenius norm of a matrix $\mathbf{X}$ is given by $\|\mathbf{X}\|_{F}^{2}=\operatorname{tr}\left(\mathbf{X}^{H} \mathbf{X}\right)=$ $\sum \sigma_{r}^{2}$, where $\sigma_{r}$ s are the singular values of $\mathbf{X}$. The weighted Frobenius norm is given by $\left\|\mathbf{X}\left(\mathbf{W}^{1 / 2}\right)^{H}\right\|_{F}$, where we have implicitly assumed that $\mathbf{X}$ has more rows than columns and $\mathbf{W}$ is a positive definite weighting matrix. For certain choices of W, the square of the weighted Frobenius norm can be thought of as a weighted sum of the singular values of $\mathbf{X}^{H} \mathbf{X}$ i.e, $\sum \alpha_{r} \sigma_{r}^{2}$. The choice of weighting matrices plays a crucial role in the effectiveness of the overall algorithm and is discussed in further detail in the next section. For a given set of weighting matrices, approximating rank using the weighted Frobenius norm leads to the following optimization problem:

$$
\begin{aligned}
\min _{\mathbf{V}} \max _{g \in\{1,2, \ldots, G\}} & \left\|\mathbf{R}_{g}\left(\left(\mathbf{W}_{g}\right)^{1 / 2}\right)^{H}\right\|_{F}^{2} \\
\text { subject to } & \mathbf{V}_{g k}(1: d, 1: d)=\mathbf{I} \forall(g, k) \\
& \mathcal{A}_{g}(\mathbf{V})=\left(\mathbf{R}_{g}\right) \forall g .
\end{aligned}
$$

Note that we implicitly assume that $\mathbf{R}_{g}$ has more rows than columns, if not we simply replace $\mathbf{R}_{g}$ with $\mathbf{R}_{g}^{H}$ in the above formulation and all subsequent steps. We assume this to ensure that the number of singular values of $\mathbf{W}_{g}$ and $\mathbf{R}_{g}$ are the same. Note that (7) is a convex optimization problem that can be transformed to a convex minimization problem through the use of dual variables, which can then be optimized using techniques such as the subgradient method. Since sub-gradient techniques are known to be slow to converge, we instead solve the following quadratic program

$$
\begin{array}{cl}
\underset{\mathbf{V}}{\operatorname{minimize}} & \sum_{g=1}^{G}\left\|\mathbf{R}_{g}\left(\left(\mathbf{W}_{g}\right)^{1 / 2}\right)^{H}\right\|_{F}^{2} \\
\text { subject to: } & \mathbf{V}_{g k}(1: d, 1: d)=\mathbf{I} \forall(g, k) \\
& \mathcal{A}_{g}(\mathbf{V})=\mathbf{R}_{g} \forall g .
\end{array}
$$

where we have replaced the maximum of a set of weighted Frobenius norms with their sum. Note that in the above quadratic program, $\mathbf{R}_{g}$ is just an auxiliary variable that can be easily eliminated and that the second set of linear constraints are straightforward to satisfy. We are thus left with a simple unconstrained quadratic program that can be optimized by solving a system of linear equations. However, replacing the min-max optimization problem with a min-sum optimization problem significantly alters the problem landscape and the ability of this reformulation to recover the desired set of beamformers depends heavily on how the weighting matrices are chosen. Nevertheless, by taking advantage of the knowledge of the achievable DoF in the network, this min-sum formulation can be effectively used to solve the min-max problem.

\section{A. Choice of Weighting Matrices}

Solving affine-constrained rank minimization by iteratively solving a series of quadratic programs that minimize a weighted Frobenius norm is first discussed in [15], where the rank of a matrix $\mathbf{X}$ is approximated using the Schatten- $p$ function given by $\operatorname{tr}\left(\mathbf{X}^{H} \mathbf{X}+\gamma \mathbf{I}\right)^{p / 2}$ for $0<p \leq 1$. Noting that the derivative of the Schatten- $p$ function is given by $p \mathbf{X}\left(\mathbf{X}^{H} \mathbf{X}+\gamma \mathbf{I}\right)^{p / 2-1}$, it is shown that the KKT conditions of the resulting affineconstrained optimization problem can be satisfied by iteratively solving a sequence of weighted-Frobenius-norm minimization problems. Mathematically, the affine-constrained rank minimization problem

$$
\begin{aligned}
\operatorname{minimize} & \operatorname{rank}(\mathbf{X}) \\
\text { subject to } & \mathcal{A}(\mathbf{X})=\mathbf{b},
\end{aligned}
$$

is solved by iteratively solving the following optimization problem:

$$
\begin{aligned}
\operatorname{minimize} & \operatorname{tr}\left(\mathbf{W}_{s} \mathbf{X}^{H} \mathbf{X}\right)=\left\|\mathbf{X}\left(\mathbf{W}_{s}\right)^{1 / 2}\right\|_{F}^{2} \\
\text { subject to } & \mathcal{A}(\mathbf{X})=\mathbf{b},
\end{aligned}
$$

where the weights $\mathbf{W}_{s}$ are updated using the update rule $\mathbf{W}_{s+1}=\left(\left(\mathbf{X}_{s}\right)^{H}\left(\mathbf{X}_{s}\right)+\gamma_{s+1} \mathbf{I}\right)^{\frac{p}{2}-1}$ where the optimal $\mathbf{X}$ obtained after the $s$ th iteration is denoted as $\mathbf{X}_{s}$ and $\gamma_{s+1}$ is the regularization parameter used in updating the $(s+1)$ th weight. When $0<p \leq 1$, the iterations of such a procedure are shown to converge. Further, the same iterative procedure can also be applied and shown to converge when $p=0$, where the weight update rule is justified by showing that it solves a fixed point equation emerging from the KKT conditions that result when rank of $\mathbf{X}$ is approximated as $\log \left(\operatorname{det}\left(\mathbf{X}^{H} \mathbf{X}+\gamma \mathbf{I}\right)\right)$.

In this paper, we set $p$ to be zero and adopt the weight update rule given above with a few important modifications. Note that when weights are updated using the update rule $\mathbf{W}_{g(s+1)}=\left(\left(\mathbf{R}_{g(s)}\right)^{H}\left(\mathbf{R}_{g(s)}\right)+\gamma_{s+1} \mathbf{I}\right)^{-1}$, the weighting matrices can be interpreted to be weighting the singular values of the matrix $\left(\mathbf{R}_{g(s)}\right)^{H} \mathbf{R}_{g(s)}$. To see this, let the singular value decomposition of $\mathbf{R}_{g(s)}$ be given by $\mathbf{P}_{g(s)} \boldsymbol{\Sigma}_{g(s)} \mathbf{Q}_{g(s)}^{H}$, then $\mathbf{W}_{g(s+1)}=\left(\mathbf{Q}_{g(s)}\right)\left(\boldsymbol{\Sigma}_{g(s)}^{2}+\gamma_{s+1} \mathbf{I}\right)^{-1}\left(\mathbf{Q}_{g(s)}\right)^{H}$. Thus, the weighting matrix $\mathbf{W}_{g(s+1)}$ imposes a penalty that is inversely proportional to the square of the magnitude of each non-zero singular value of $\mathbf{R}_{g(s)}$. Since small, non-zero singular values are heavily penalized, the iterative procedure is incentivized to reduce them to zero, thus reducing the rank of $\mathbf{R}_{g(s)}$.

The formulation in (8) does not incorporate any information on the expected rank of the interference matrices and a direct application of the weighting procedure outlined above is unlikely to generate the requisite number of interferencefree dimensions due to the presence of many local minima. Further the formulation in (8) is not inherently inclined to generate the same number of interference free dimensions at each BSs, thereby leading to scenarios where we have more 
than necessary number of interference free dimensions at some BSs and insufficient interference-free dimensions at other BSs. The key observation of this paper is that we can modify the weight update rule to take advantage of the fact that we know how many DoF are achievable per user in the network and to ensure that we obtain the requisite number of interference-free dimensions at each BS in a balanced manner.

Since we are looking for transmit beamformers that ensure $\operatorname{rank}\left(\mathbf{R}_{g}\right) \leq N-K d \forall g$, we require $z=$ $\min (K d,(G K d-N))$ singular values of $\mathbf{R}_{g}$ to be zero. To avoid local minima where rank of $\mathbf{R}_{g}$ is not sufficiently minimized, we couple the penalties associated with each of the $z$ smallest singular values of $\mathbf{R}_{1}, \mathbf{R}_{2}, \ldots, \mathbf{R}_{G}$. Let $\left\{\sigma_{g r}\right.$ : $r=1,2, \ldots, \min ((G-1) K d, N)\}$ be the set of singular values of $\mathbf{R}_{g}$ obtained after the sth iteration, ordered in the descending order i.e., $\sigma_{g r} \geq \sigma_{g(r+1)}$. Further, let $\sigma_{\min }^{2}=\min _{g, r} \sigma_{g r}^{2}$ and define the diagonal matrix $\mathbf{D}_{g(s)}=$ $\operatorname{diag}\left([\sigma_{g 1}^{2}, \sigma_{g 2}^{2}, \ldots, \sigma_{g(N-K d)}^{2}, \underbrace{\sigma_{\min }^{2}, \sigma_{\min }^{2}, \ldots, \sigma_{\min }^{2}}_{\mathrm{z} \text { times }}]\right)$. We set the weights for the $(s+1)$ th iteration to be $\mathbf{W}_{g(s+1)}=$ $\left(\mathbf{Q}_{g(s)} \mathbf{D}_{g(s)}\left(\mathbf{Q}_{g(s)}\right)^{H}+\gamma_{s+1} \mathbf{I}\right)^{-1}$. Such an update equally penalizes each of the $z$ smallest singular values of $\mathbf{R}_{i}$, thereby encouraging the algorithm to seek aligned beamformers where all $z$ smallest singular values can be simultaneously set to zero. The proposed iterative procedure can be summarized as follows:

1) Initialize $\mathbf{W}_{g(1)}=\mathbf{I} \forall g$, set $\gamma=\gamma_{1}, s=1$.

2) Iterate over $s$ :

a) Solve (8) using weights $\mathbf{W}_{g(s)}$ and denote the optimal interference matrices as $\mathbf{R}_{g \text { opt }}$.

b) Compute the reduced SVD of $\mathbf{R}_{g o p t}$, and denote it as $\mathbf{P}_{g(s)} \boldsymbol{\Sigma}_{g(s)}\left(\mathbf{Q}_{g(s)}\right)^{H}$.

c) Set $\sigma_{\text {min }}^{2}=\min _{g, r} \sigma_{g r}^{2}$, where $\sigma_{g r}$ are the singular values of $\mathbf{R}_{g(s)}$ arranged in descending order.

d) Set $\quad \mathbf{D}_{g(s)}=\operatorname{diag}\left(\left[\sigma_{g 1}^{2}, \ldots, \sigma_{g(N-K d)}^{2}\right.\right.$, $\underbrace{\sigma_{\min }^{2}, \ldots, \sigma_{\min }^{2}}_{\mathrm{z} \text { times }}])$.

e) Update $\gamma$.

f) Update $\mathbf{W}_{g(s+1)}=\left(\mathbf{Q}_{g(s)} \mathbf{D}_{g(s)}\left(\mathbf{Q}_{g(s)}\right)^{H}+\gamma \mathbf{I}\right)^{-1}$.

g) Return to Step (2a) if $s<$ iter $_{\max }$.

The parameter $\gamma$ acts as a regularization constant that makes sure the weighting matrices are positive definite. Further, $\gamma$ determines the penalty imposed on small non-zero singular values. Typically, $\gamma$ is adaptively reduced with each iteration to prevent the algorithm from prematurely converging to local minima that do not completely align interference. As suggested in [12], we set $\gamma$ in the $k$ th iteration to be $\gamma^{k}=\frac{1}{(1+\delta)^{k}}$, where $\delta$ is a small positive constant.

When weights are updated according to the original update rule given in [12], the algorithm can be shown to be converge. For the weight update rule proposed in this paper, no such convergence guarantees exist. Hence, we run the proposed algorithm for a fixed number of iterations $\left(i t e r_{\max }\right)$. Once the transmit beamformers in the uplink are designed, the receiver beamformers at the $g$ th BS to recover the data streams of the $k$ th user can be chosen to be the left-singular vectors

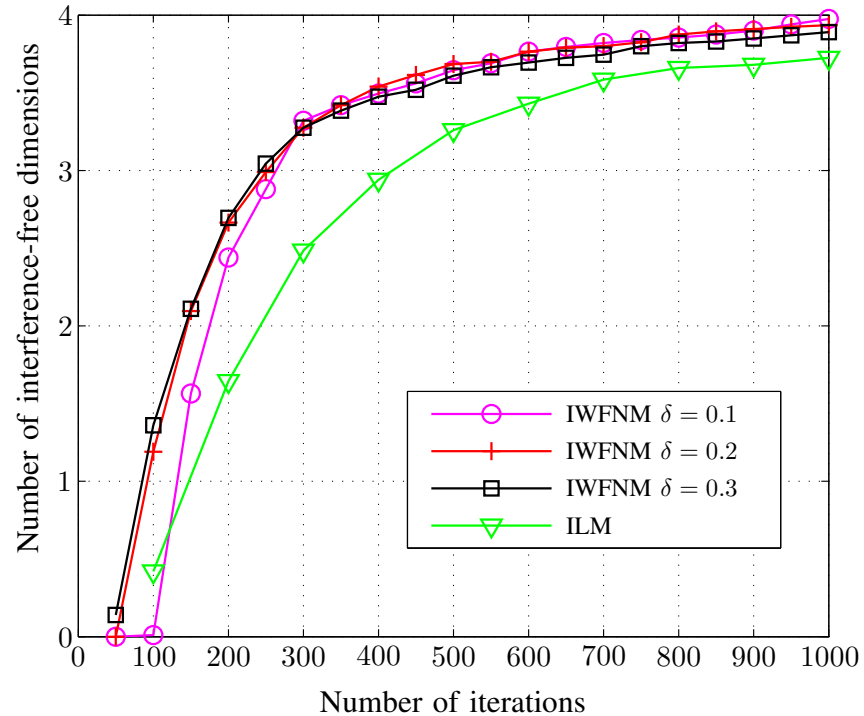

Fig. 1. Interference-free dimensions as a function of iterations for a $(4,1,2,3)$ network with 1 data stream per user

corresponding to the $d$ smallest singular values of the matrix $\mathbf{R}_{g}$ augmented with the interfering vectors from other users in the same cell.

\section{Simulation Results}

In order to test our algorithm, we first consider a $(4,1,2,3)$ interference channel with one data stream per user. This system is known to be a proper and feasible system [13]. For perfect interference alignment, we need interfering vectors to occupy two or fewer dimensions at every receiver. Note that this system is on the proper-improper boundary and has no redundant variables. We compare the proposed iterative weighted-Frobeniusnorm minimization (IWFNM) algorithm with the interference leakage minimization (ILM) algorithm proposed in [3]. One iteration of ILM requires computing $(G(K+1))$ eigenvalue decompositions to update the transmit and receive beamformers. One iteration of IWFNM requires solving an unconstrained quadratic program involving $G K(M-d) d$ variables and $G$ singular value decompositions to compute the weighting matrices. The convex optimization problem in (8) can be solved analytically, or by using CVX, a package for specifying and solving convex programs [19], [20]. The algorithms are tested over 200 channel realizations with channel coefficients drawn i.i.d from $\mathcal{C N}(0,2)$. The algorithms are run for a fixed number of iterations, and the interference-free dimensions at BS $g$ are counted as the number of singular values of $\mathbf{U}_{g k}^{H} \mathbf{H}_{g k, g} \mathbf{V}_{g k}>$ $10^{-2}$ for all $g$ while subtracting the number of singular values of $\mathbf{U}_{g k}^{H}\left[\mathbf{R}_{g}, \mathbf{H}_{(g 1, g)} \mathbf{V}_{g 1}, \ldots, \mathbf{H}_{(g(k-1), g)} \mathbf{V}_{g(k-1)}\right.$, $\left.\mathbf{H}_{(g(k+1), g)} \mathbf{V}_{g(k+1)}, \ldots, \mathbf{H}_{(g K, g)} \mathbf{V}_{g K}\right]>10^{-5}$.

The simulation results are plotted in Fig. 1, where the number of interference-free dimensions for the overall network averaged over all the channel realizations are plotted as a function of the number of iterations. We opt to show the number of interference-free dimensions rather than systemlevel performance metrics such as sum-rate because the former is more directly related to the optimization objective of this 


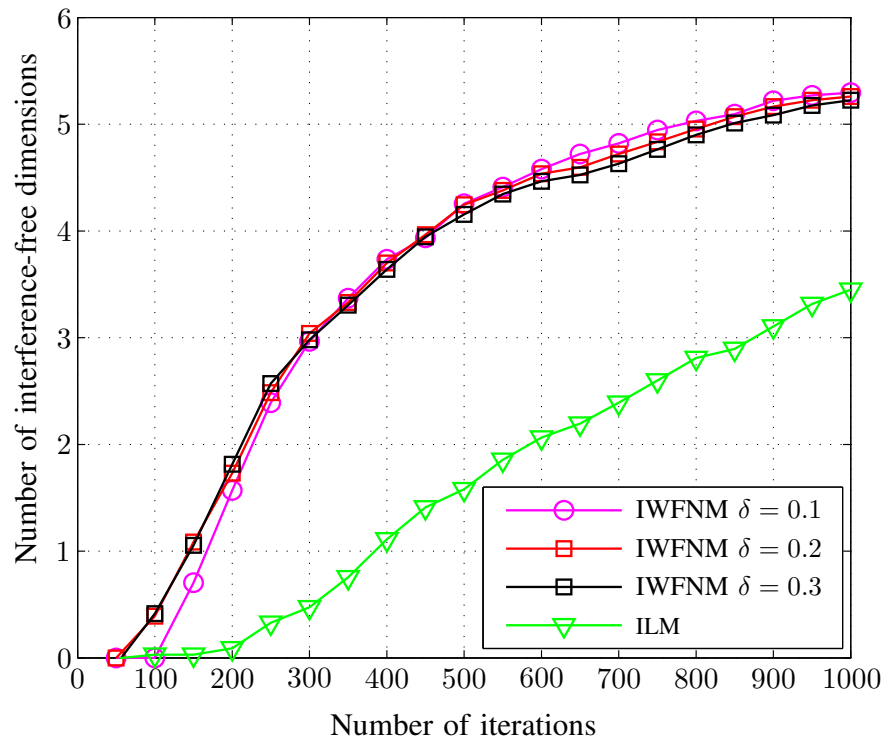

Fig. 2. Interference-free dimensions as a function of iterations for a $(3,2,2,5)$ network with 1 data stream per user.

paper. For IWFNM, the parameter $\delta$ was varied from 0.1 to 0.3 . It can be seen that for most channel instances the IWFNM algorithm is able to construct four interference free dimensions within a few hundred iterations. Further, when compared to ILM, IWFNM shows faster convergence, requiring hundreds of fewer iterations to generate the same number of interference free dimensions. Also note that the parameter $\delta$ has only a negligible impact on the performance of the algorithm.

In Fig. 2 we consider the $(3,2,2,5)$ cellular network with the goal of achieving $1 \mathrm{DoF} / \mathrm{user}$. This system is also on the properimproper boundary and is known to be feasible. Once again we see that although IWFNM shows better performance than ILM, converging to beamformers that perfectly align interference at all terminals (6 in this network) takes significantly more number of iterations. Even after about 1000 iterations, in only $25-30 \%$ of the channel instances is the interference completely aligned.

Drawing upon insights from compressed sensing, as both ILM and IWFNM approximate rank as a weighted $\ell_{2}$ norm rather than using the $\ell_{1}$ norm, it is not surprising that ILM and IWFNM take several hundred iterations to converge to aligned solutions. While the use of weighted $\ell_{1}$ norm is better suited to generate a sparse set of singular values, such an approximation leads to semidefinite programs that are significantly more complex to solve [8], [9], [11]. This poses an interesting tradeoff between complexity per iteration and the total number of iterations that is difficult to resolve except through simulations.

\section{CONCLUSION}

In this paper we propose an iterative weighted-Frobeniusnorm minimization algorithm to design beamformers for linear interference alignment in MIMO cellular networks. Each iteration of this algorithm requires solving a simple quadratic program that minimizes the weighted Frobenius norm of an interference matrix. A novel weight update rule is proposed that steers the algorithm towards aligned solutions and thereby generating the requisite number of interference free dimensions. The algorithm shows improved convergence when compared to algorithms of similar complexity such as interference leakage minimization.

\section{REFERENCES}

[1] V. R. Cadambe and S. A. Jafar, "Interference alignment and degrees of freedom of the K-user interference channel," IEEE Trans. Inf. Theory, vol. 54, no. 8, pp. $3425-3441$, Aug. 2008.

[2] A. S. Motahari, S. O. Gharan, and A. K. Khandani, "Real interference alignment with real numbers." [Online]. Available: http: //arxiv.org/abs/0908.1208

[3] K. Gomadam, V. R. Cadambe, and S. A. Jafar, "A distributed numerical approach to interference alignment and applications to wireless interference networks," IEEE Trans. Inf. Theory, vol. 57, no. 6, pp. 3309-3322, Jun. 2011.

[4] S. W. Peters and R. W. Heath, "Interference alignment via alternating minimization," in IEEE Int. Conf. Acoust., Speech Signal Process., Apr. 2009, pp. 2445-2448.

[5] B. Zhuang, R. A. Berry, and M. L. Honig, "Interference alignment in MIMO cellular networks," in IEEE Int. Conf. Acoust., Speech Signal Process., May 2011.

[6] D. S. Papailiopoulos and A. G. Dimakis, "Interference alignment as a rank constrained rank minimization," IEEE Trans. Signal Process., vol. 60, no. 8, pp. 4278-4288, Aug. 2012.

[7] D. A. Schmidt, C. Shi, R. A. Berry, M. L. Honig, and W. Utschick, "Minimum mean squared error interference alignment," in Asilomar Conf. Signals, Syst. Comput., Nov. 2009, pp. 1106-1110.

[8] H. Du and T. Ratnarajah, "On the nuclear norm approach to interference alignment," in Asilomar Conf. Signals, Syst. Comput., Nov. 2012, pp. $1571-1575$.

[9] H. Du, T. Ratnarajah, M. Sellathurai, and C. Papadias, "Reweighted nuclear norm approach for interference alignment," IEEE Trans. Commun., vol. 61, no. 9, pp. 3754-3765, Sep. 2013.

[10] C. Wang, T. Gou, and S. A. Jafar, "Subspace alignment chains and the degrees of freedom of the three-user MIMO interference channel," IEEE Trans. Inf. Theory, submitted for publication. [Online]. Available: http://arxiv.org/abs/1109.4350

[11] G. Sridharan and W. Yu, "Interference alignment using reweighted nuclear norm minimization," in IEEE Int. Conf. Acoust., Speech Signal Process., May 2013.

[12] K. Mohan and M. Fazel, "Iterative reweighted algorithms for matrix rank minimization," Journal of Machine Learning Research, vol. 13, no. 1, pp. 3441-3473, 2012.

[13] C. M. Yetis, T. Gou, S. A. Jafar, and A. H. Kayran, "On feasibility of interference alignment in MIMO interference networks," IEEE Trans. Signal Process., vol. 58, no. 9, pp. 4771-4782, Sep. 2010.

[14] M. Razaviyayn, G. Lyubeznik, and Z.-Q. Luo, "On the degrees of freedom achievable through interference alignment in a MIMO interference channel," IEEE Trans. Signal Process., vol. 60, no. 2, pp. 812 -821, Feb. 2012.

[15] K. Mohan and M. Fazel, "Iterative reweighted least squares for matrix rank minimization," in Annu. Allerton Conf. Commun., Control, Computing, Sep. 2010, pp. 653-661.

[16] M. Fazel, H. Hindi, and S. P. Boyd, "Log-det heuristic for matrix rank minimization with applications to Hankel and Euclidean distance matrices," in Proceedings of the American Control Conference, vol. 3, Jun. 2003, pp. 2156-2162.

[17] — "Rank minimization and applications in system theory," in Proceedings of the American Control Conference, vol. 4, Jun. 2004, pp. 3273-3278

[18] K. Mohan and M. Fazel, "Reweighted nuclear norm minimization with application to system identification," in Proc. American Control Conf., Jun. 2010, pp. 2953-2959.

[19] CVX Research, Inc., "CVX: Matlab software for disciplined convex programming, version 2.0 beta," September 2012. [Online]. Available: http://cvxr.com/cvx

[20] M. Grant and S. Boyd, "Graph implementations for nonsmooth convex programs," in Recent Advances in Learning and Control, ser. Lecture Notes in Control and Information Sciences, V. Blondel, S. Boyd, and H. Kimura, Eds. Springer-Verlag Limited, 2008, pp. 95-110. [Online]. Available: http://stanford.edu/ boyd/graph_dcp.html 